\title{
Choroidal lesions in patients with AIDS
}

\author{
Marc A Rosenblatt, Carol Cunningham, Steven Teich, Alan H Friedman
}

\begin{abstract}
Seven cases of bilateral, scattered, yellowwhite choroidal lesions have been seen in AIDS patients since January 1988. One resulted from presumed extension of cryptococcal meningitis into the optic nerve and choroid. All the remaining six patients had pneumocystis pneumonia at some time during the course of the disease and were receiving aerosolised pentamidine therapy. None died quickly of disseminated Pneumocystis carinii infection, unlike previously reported patients. Mycobacterial infection was also present in five of these six patients. The differential diagnosis of this entity in AIDS patients is discussed.
\end{abstract}

Scattered choroidal yellow-white lesions in AIDS patients have recently been described as a marker for disseminated and rapidly fatal Pneumocystis carinii infection.' We present a series of seven cases with similar appearances but varied outcomes. Possible alternative aetiologies are discussed.

\section{Case reports}

\section{CASE 1}

A 31-year-old homosexual male with AIDS had four previous episodes of Pneumocystis carinit pneumonia and one of cytomegalovirus (CMV) pneumonia. Mycobacterium avium intracellulare infection had been noted on endobronchial biopsy. Medications included isoniazid, rifampicin, ganciclovir, and aerosolised pentamidine. During recurrence of pneumonia, which was without visual symptoms and with norma acuity, examination with dilated pupils revealed bilateral, flat, yellow-white choroidal lesions through all quadrants, varying in size from 0.25 to 2 disc diameters. Rapid plasma reagin, toxoplasmosis immunofluorescence antibody test, and serological tests for histoplasmosis, coccidioidomycosis, and cryptococcus were negative. After three weeks of intravenous pentamidine and a reinduction course of ganciclovir the pneumonia cleared. After two months the choroidal lesions became more discrete and orange, with overlying pigment proliferation. He died of staphylococcal septicaemia 11 months later without necropsy.

M A Rosenblatt

$S$ Teich

A H Friedman

Department of Ophthalmology, New

York University School of Medicine, New York CCunningham

Correspondence to: M A Rosenblatt, MD, 9 East 75th Street, New York, NY 10021, USA.

Accepted for publication 11 May 1990
20 choroidal lesions in each eye while in hospital in June 1988 for cryptococcal meningitis with bone marrow involvement and increased intracranial pressure. Despite treatment with intravenous amphotericin, palsies of cranial nerves
III, V, VI, and VII developed. The optic disc was swollen, with dilated retinal venules and peripapillary haemorrhages.

Computerised tomography revealed enlarged optic nerves. Despite treatment with flucytosine and intravenous dexamethasone, vision deteriorated to finger counting in both eyes after one month. An electroretinogram (ERG) was normal, but visually evoked potential (VEP) was unrecordable. Fluorescein angiography revealed diffuse disc leakage and hypofluorescence corresponding to the choroidal lesions. Visual loss was thought to be due to cryptococcal invasion of the optic nerves.

CASE 3

A 28-year-old homosexual male with AIDS had multiple episodes of $P$ carinii pneumonia, after which he received aerosolised pentamidine. CMV retinitis of the right eye treated with ganciclovir progressed to a retinal detachment. Isoniazid, rifampicin, and ethambutol were prescribed for bone marrow granulomas. Subretinal creamy yellow-white lesions appeared about the macula in the left eye. Over two weeks similar lesions appeared under the bullous detachment in the right eye, and new lesions appeared in the left eye. Vision remained 20/20 in the left eye. Fever developed. Serological testing for rapid plasma reagin, toxoplasmosis, cryptococcus, coccidiomycosis titres, adenovirus titres, and Epstein-Barr virus titres were negative. A gallium scan showed increased uptake in the mediastinum and retroperitoneal lymph nodes. Intravenous pentamidine was begun for presumed disseminated $P$ carinii infection. The choroidal lesions become more discrete and orange, with pigment proliferation at their edges. The patient returned home on oral dapsone prophylaxis for $P$ carinii pneumonia (PCP), and one month later his local ophthalmologist noted no evidence of choroidal lesions in either eye. His visual acuity was light perception OD and 20/20 OS.

\section{CASE 4}

A 37-year-old intravenous drug user with AIDS, maintained on aerosolised pentamidine after five bouts of $P$ carinii pneumonia, had 20/40 vision OD while in the hospital for bronchoscopyproven pneumocystis pneumonia. Amsler grid testing revealed a large paracentral scotoma OD. Examination under dilated pupils showed a fivedisc diameter, flat, grey-yellow choroidal lesion subfoveally with a brighter area as its nasal edge, suggesting more acute inflammation. Ten to 15 smaller choroidal lesions were scattered outside the posterior pole. In the left eye there were at least 20 similar choroidal lesions. The pneumonia improved on trimetrexate and the lesions became 


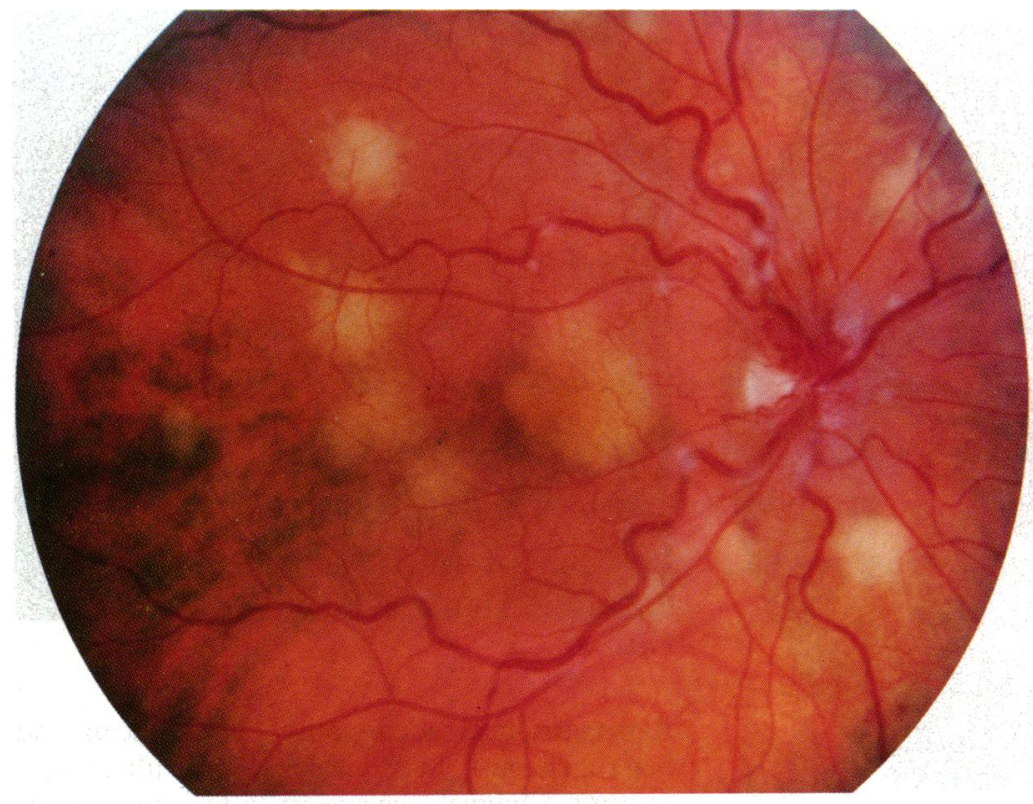

Figure $1 A$

Figure I Case 6. A: Right eye: Scattered lesions with one under the fovea and flame haemorrhages at the disc margin with several cotton-wool spots and haemorrhages. Visual acuity 20/80. B: Left eye: similar retinal lesions on presentation. C: Fluorescein angiogram right eye, early arteriolar phase showing hypofluorescence of lesions. D: Fluorescein angiogram right eye, hyperfluorescence later in sequence.

more distinct. Serological testing for syphilis (VDRL), cryptococcal antigen, and toxoplasmosis gave negative results. A diagnosis of presumed disseminated $P$ carinii infection was made and the patient put on prophylactic therapy with oral dapsone. A splenectomy three months later for idiopathic thrombocytic purpura revealed both $P$ carinii and acid-fast bacteria. Isoniazid and ethambutol were added to the treatment. A year later, during the seventh admission to hospital for $P$ carinii pneumonia, acuity and Amsler grid results had returned to normal and the choroidal lesions had become deep orange.

CASE 5

A 42-year-old AIDS patient had three recur-

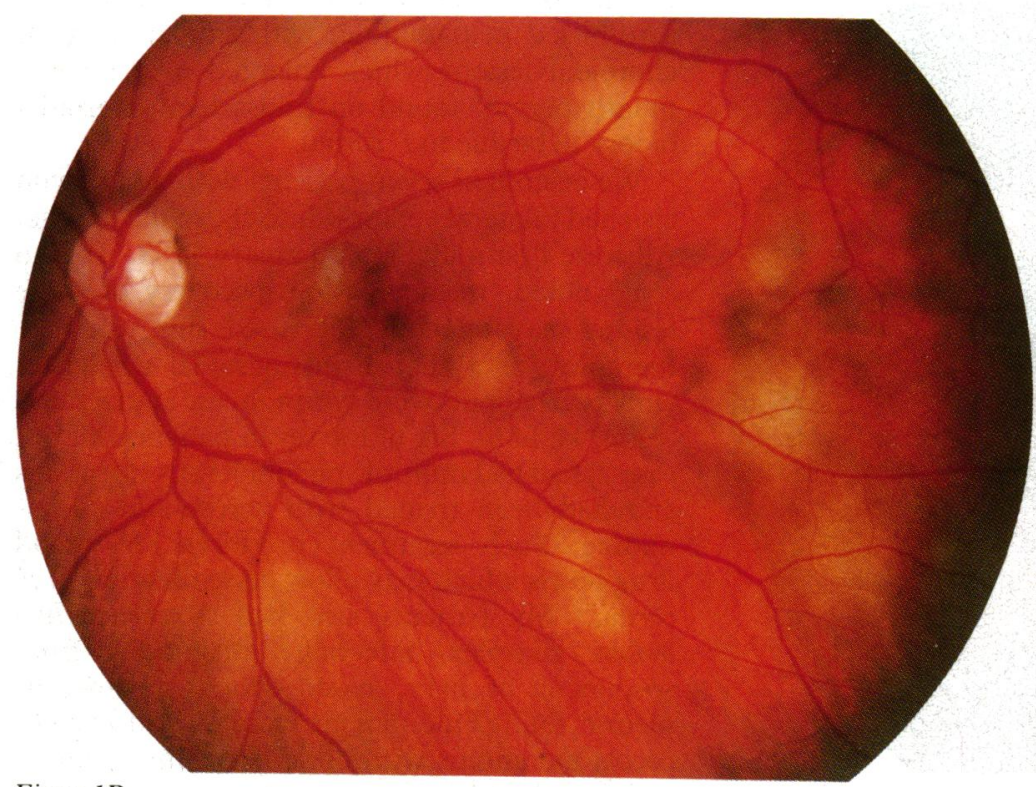

Figure $1 B$

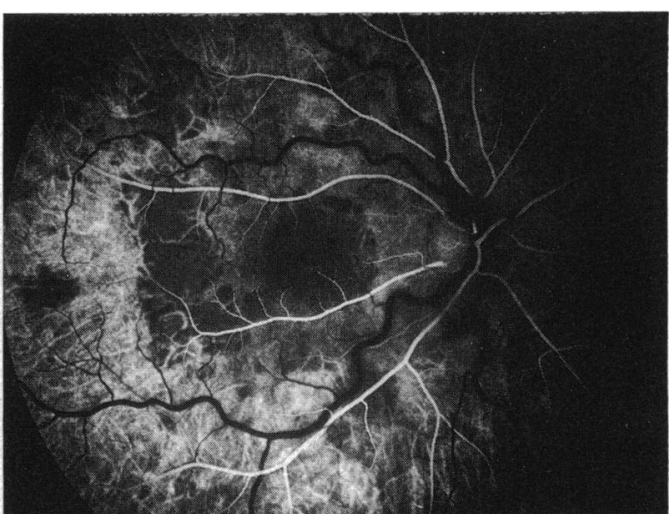

Figure 1C

rences of PCP in two years; he was taking rifampicin and isoniazid for Mycobacterium avium intracellulare infection, acyclovir for herpes simplex, aerosolised pentamidine for PCP, and azathioprine. CMV retinitis was found in the left eye, reducing vision to 20/400. After treatment with ganciclovir for three months his acuity was unchanged, but he developed grey-yellow lesions throughout the right fundus at the level of the retinal pigment epithelium. Similar lesions were seen in the non-atrophic areas of the left fundus. Fluorescein angiography revealed early blockage and late staining of the lesions.

\section{CASE 6}

A 37-year-old male with AIDS was admitted to hospital in September 1988 for bilateral pleural effusions after eight months of aerosolised pentamidine. His visual acuity was $20 / 20$ OU. Examination under dilated pupils revealed round and oval yellow-white choroidal lesions 0.5 to 2 disc diameters scattered in the posterior pole with one underlying the fovea OD. There were flame haemorrhages on and near the disc OD (Figs 1A, B). Fluorescein angiography revealed blockage early and late staining of the lesions (Figs 1C, D). A pleural tap revealed acid-fast bacilli; the patient's vision declined to $20 / 80$ in the right eye. Isoniazid and rifampicin were begun as well as intravenous pentamidine. Two months later the visual acuity returned to 20/20 OD. Cultures grew Mycobacterium avium intracellulare from the pleural fluid, but cerebrospinal fluid (CSF) cultures were negative. By January 1989, four months after the initial examination, the lesions had totally resolved.

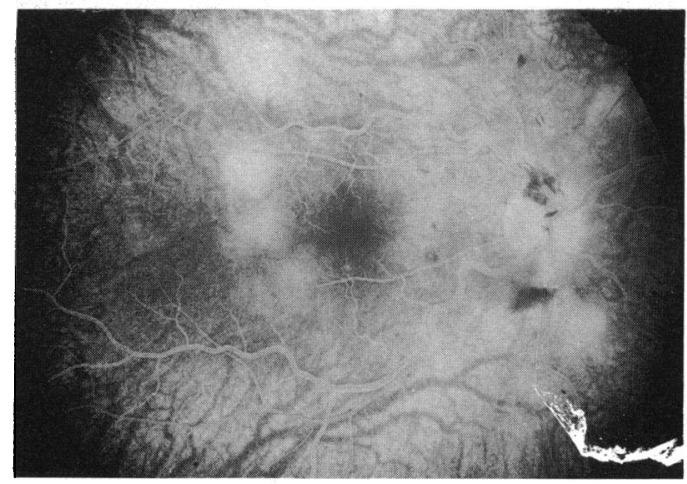

Figure 1D 


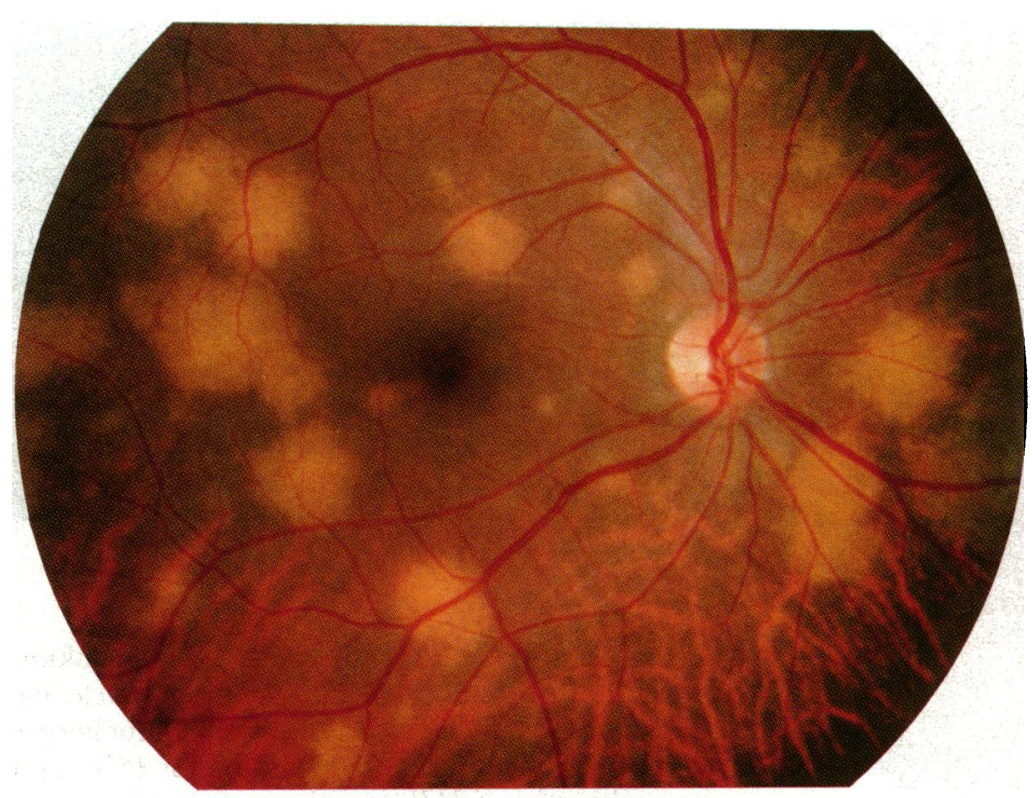

Figure $2 A$

Figure 2 Case 7: A: Right eye: deep yellow lesions on presentation. B: Left eye: subfoveal lesions with 20/20 visual acuity. C: Midphase fluorescein angiogram right eye, hyperfluorescence of lesions.

CASE 7

A 40-year-old AIDS patient had pneumocystis pneumonia three times treated with cotrimoxazole, intravenous pentamidine, and maintenance aerosolised pentamidine. On routine screening we found $20 / 20$ vision $O U$ and numerous grouped, yellow, deep round lesions in both eyes scattered about the posterior pole, some coalescing into larger oval lesions (Figs 2A, B). One lesion was directly subfoveal in the left eye. Fluorescein angiography revealed early blockage and late leakage (Fig 2C). Rapid plasma reagin, haemagglutination assay test for syphilis, and CMV titres were negative. The CSF was normal, with negative results to cryptococcal antigen and VDRL tests. The patient was anergic to skin testing. Vision remained stable, but the patient developed unexplained fevers.

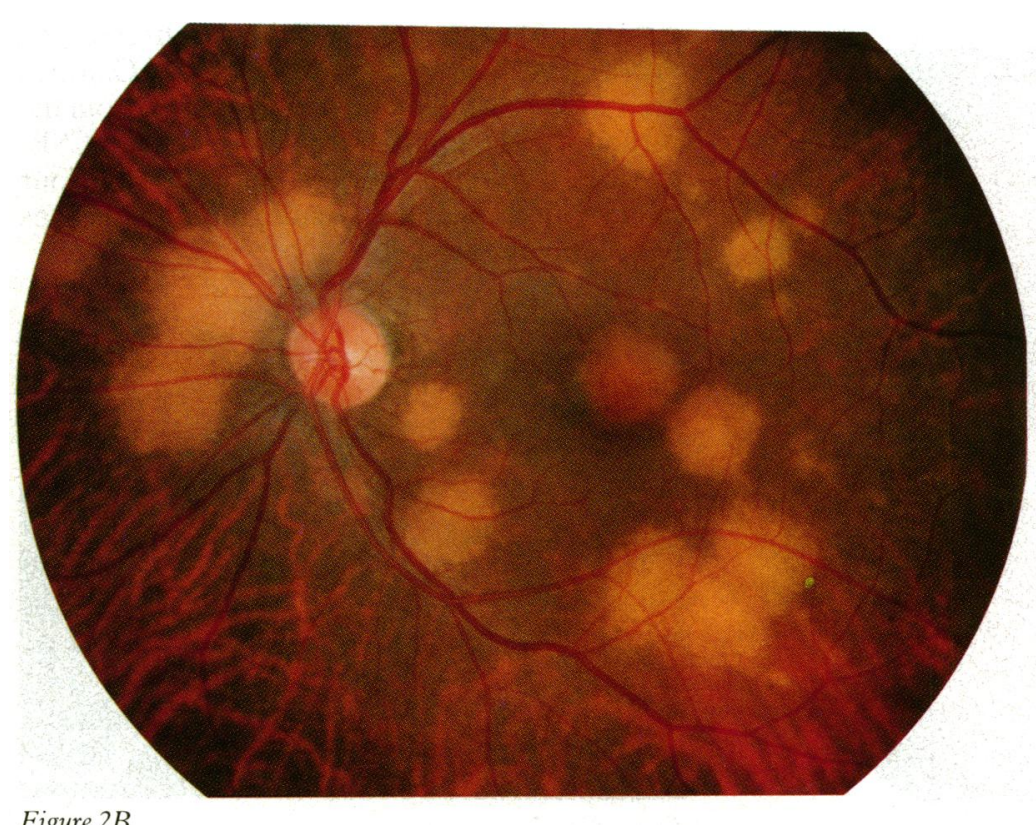

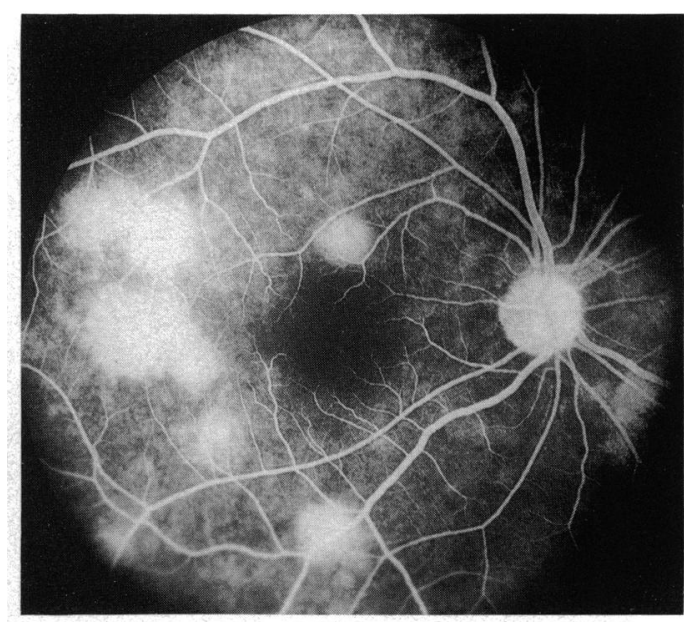

Figure 2C

Retroperitoneal adenopathy on CT scan and heterogeneous liver lesions on ultrasound prompted biopsy; the specimen was positive for acid-fast bacilli. Bone marrow aspirate was hypocellular, and acid-fast bacilli were not seen. Treatment was begun with isoniazid, rifampicin, pyrazinamide, and streptomycin. Six months after the initial findings, the visual acuity was stable and the lesions were fading.

\section{Discussion}

Choroidal lesions have occasionally been reported in AIDS patients. Macher et al described the histopathology of a case of yellow choroidal lesions secondary to $P$ carinii infection. ${ }^{2}$ More recently Rao et al reported three cases of yellowwhite choroidal plaques which, on necropsy, revealed trophozoites of $P$ carinii. ${ }^{\prime}$ Visual acuity was decreased in one eye from accompanying CMV retinitis and reported as 20/30 and 20/60 in two other eyes. All the patients had received aerosolised pentamidine prophylaxis for $P$ carinit pneumonia and all died within three weeks of discovery of the retinal lesions. The authors concluded that such fundus findings allow a presumptive diagnosis of disseminated $P$ carinii disease and warrant agressive chemotherapy. Freeman et al described an AIDS patient, also on aerosolised pentamidine, with similar bilateral choroidal lesions which enlarged over a fourmonth period, found on transscleral choroidal biopsy to be due to $P$ carinii. ${ }^{3}$

We examined seven patients with AIDS who also had bilateral yellow-white choroidal lesions. All were first noted after January 1988. As Rao et $a l^{1}$ noted, the increasing discovery of these lesions may relate to increased survival, new therapies, or a change in microbial virulence. Only two patients were symptomatic at diagnosis (cases 4 and 6). Six of the seven patients (all but case 2) had had multiple pneumocystis infections and had been treated with aerosolised pentamidine. Such treatment produces low blood drug levels which may allow dissemination of $P$ carinii. ${ }^{4}$ Six patients also had Mycobacterium avium complex infections diagnosed from specimens of liver, blood, bone marrow, or pleural fluid. One patient (case 2) had cryptococcal meningitis with optic nerve involvement and died, blind, in four months. Another patient 
died after 15 months, without necropsy. In three patients (cases 3, 4, and 6) the lesions disappeared in three to six months during systemic treatment for PCP with intravenous trimetrexate or pentamidine followed by oral dapsone. The lesions remained unchanged in two of the patients after three to six months of follow-up. All our patients except number 2 are thought to represent cases of choroiditis due to disseminated $P$ carinii infection. $P$ carinii was demonstrated histologically in the spleen of patient 4 . The clinical appearance of our cases is identical to that of the four previously reported cases.

Interestingly, these six patients also had disseminated mycobacterial infection, an entity whose frequent bacteraemias might account for seeding of the vascular choroid. ${ }^{56}$ Little local tissue destruction and inflammation are associated with $M$ avium infection, a fact compatible with normal acuity despite subfoveal lesions. ${ }^{78}$ $M$ avium has been cultured from one retina and choroid of an AIDS patient by Pepose et al; choroidal granulomas were found in $6 \%$ of their series.' Intracellular acid-fast bacilli in two choroidal granulomas were also seen at necropsy by Holland et al. ${ }^{10}$ Choroidal mycobacterial disease in AIDS patients has also been described elsewhere. ${ }^{112}$ The appearance of our patients, their use of aerosol pentamidine, and response or lack of progression with systemic antipneumocystis therapy leads us to believe that $P$ carinii is more likely to be the primary infection in our six cases; however, coinfection with mycobacteria is possible. In the absence of reliable skin testing or antibiotic agents that could be used in a therapeutic trial, the diagnosis of $M$ avium choroiditis can be made only on biopsy. ${ }^{13}$ Since our patients generally had good vision, biopsy was not recommended. Because no cure for $M$ avium infection exists, and there is no diagnostic test other than biopsy for its presence in the eye, the role of a thorough investigation for systemic atypical mycobacteria in such patients is unclear.

Patient 2 differed from our other patients. $\mathrm{He}$ had cryptococcal meningitis with bilateral optic neuropathy; choroidal lesions probably also represented cryptococcal infection. A similar case was described by Winward et al. ${ }^{14}$ Cryptococcal choroiditis has been well documented in patients with and without AIDS. ${ }^{14-19}$

Other causes of choroidal lesions in AIDS patients must also be considered. Secondary syphilis can present as a multifocal choroiditis with large placoid foveolar lesions. ${ }^{20-22}$ None of our patients had reactive rapid plasma reagin (RPR) of blood or CSF, but falsely negative serology can occur in AIDS patients. ${ }^{23-25}$ Bilateral histoplasma chorioretinitis has been reported in an AIDS patient, but the clinical picture differed from that of our cases. ${ }^{26}$

Reticulum cell sarcoma can present with isolated multicentric choroidal lesions. Central nervous system lymphoma is common in AIDS patients, but choroidal involvement has not yet been reported. ${ }^{27}$ Septic choroiditis can start as a multifocal choroiditis but would be expected to progress to uveitis and panophthalmitis. ${ }^{28}$ Choroidal lesions can also be caused by metastatic carcinoma, sarcoidosis, Vogt-
Koyanagi-Harada syndrome, or sympathetic ophthalmia. ${ }^{29-31}$

We have been impressed by two aspects of the choroiditis in our patients. Many lesions caused little or no visual problem even with the fovea directly involved and were reversible to some degree with therapy. Our only patient with severe visual loss had cryptococcal choroiditis with optic neuropathy.

Secondly, the finding of presumed $P$ carinii choroiditis in an AIDS patient does not necessarily imply death within a few weeks as previously reported. None of our patients died precipitously; most survived several months after rapid systemic treatment for $P$ carinii. Increased awareness of this marker for disseminated pneumocystis infection may allow for its earlier detection and treatment. Diagnostic imaging may reveal sites of disease amenable to biopsy, confirming dissemination of disease. Disseminated $P$ carinii infection requires systemic treatment, probably on a long-term basis. Future investigation of ocular involvement in the beige mouse model of $M$ avium infection may be helpful. ${ }^{32}$

1 Rao NA, Zimmerman PL, Boyer D, et al. Study of Pneumocystis carinii choroiditis. Am $\mathcal{F}$ Ophthalmol 1989; 107 218-28.

2 Macher AM, Bardenstein DS, Zimmerman L. $P$ carini choroiditis in a male homosexual with AIDS and disseminated pulmonary and extrapulmonary $P$ carinii infection. nated pulmonary and extrapulm

3 Freeman WR, Gross JG, Labelle J, Oteken K, Katz B, Wiley CA. Pneumocystis carinii choroidopathy: a new clinical CA. Pneumocystis carinii choroidopathy:

4 Raviglione MC, Garner GR, Mullen MP. Pneumocystis carini in bone marrow. Ann Intern Med 1988; 109: 253

5 Macher AM, Kovacs JA, Gill V, Roberts GD. Bacteremia due to Mycobacterium avium intracellulare in the acquired immunodeficiency syndrome. Ann Intern Med 1983; 99. 782-5

6 Wong B, Edwards FF, Kiehn TE, et al. Continuous high grade Mycobacterium avium-intracellulare bacteremia in patients with the acquired immune deficiency syndrome. Am F Med 1985; 78: 35-40.

7 Wallace JM, Hannah JB. $M$ avium complex infection in patients with AIDS. Chest 1988; 93: 926-32.

8 Agins B, Berman DS, Spicehandler D, EllSadr W, Simberkoff MS, Rahal JKJ. Effect of combined therapy with ansamycin, clofazimine, ethambutol, and isoniozid for Mycobacterium avium infection in patients with AIDS. F Infect Dis 1989; 4: 784-7.

9 Pepose JS, Holland GN, Nestor MS. Acquired immune deficiency syndrome. Ophthalmology 1985; 92: 472-84.

10 Holland GN, Pepose JS, Pettit TH. Acquired immune deficiency syndrome: ocular manifestations. Ophthalmology 1983; 90: 859-73.

11 Blodi BA, Johnson MW, McLeish WM, Gass JDM. Presumed choroidal tuberculosis in an HIV infected host. $A m \mathcal{F}$ Ophthalmol 1989; 108: 605-7.

12 Croxalto JO, Mestre C, Puente S, Gonzalez G. Nonreactive tuberculosis in a patient with acquired immune deficiency syndrome. Am $\mathcal{F}$ Ophthalmol 1986; 102: 659-60.

13 Abrams J, Schlaegel TF. The role of the isoniazid therapeutic test in tuberculous uveitis. Am 7 Ophthalmol 1982; 94: 511-5

14 Winward KE, Hamed LM, Glaser JS. The spectrum of optic nerve disease in human immunodeficiency virus infection. Am $\mathcal{F}$ Ophthalmol 1989; 107: 373-80.

15 Kupfer C, McCrane E. A possible cause of decreased vision in cryptococcal meningitis. Invest Ophthalmol Vis Sci 1974; 13: $801-4$.

16 Okun E, Butler WT. Ophthalmologic complications of cryptococcal meningitis. Arch Ophthalmol 1961; 71:96-101.

17 Bisseru B, Bajaj A, Carruthers RH, Chhabra H. Pulmonary and bilateral retinochoroidal cryptococcosis. Br f Ophthal mol 1983; 67: 157-61.

18 Shields JA, Wright DM, Augsberger JJ, Wolkowicz MI Cryptoccocal chorioretinitis. Am $\mathcal{F}$ Ophthalmol 1980; 89:

19 Hiles DA, Font RL. Bilateral intraocular cryptococcosis with unilateral spontaneous regression. Am f Ophthalmol 1968 : 65: 98-108.

20 Halperin LS, Lewis H, Blumenkranz MS, Gass JDM, Olk R, Fine $S$. Choroidal neovascular membrane and other chorioretinal complications of acquired syphilis. Am $\mathcal{F}$ Ophthalmo 1989; 108: 554-62.

21 Schlaegel TF, Kao SF. A review of 28 presumptive cases of syphilitic uveitis. Am f Ophthalmol 1982; 93: 412-4.

22 Ross WH, Sutton HFS. Acquired syphilitic uveitis. Arch Ophthalmol 1980; 98: 496-8. 
23 Kleiner RC, Najarian L, Levenson J, Kaplan HJ. AIDS complicated by syphilis can mimick uveitis and Crohn's disease. Arch Ophthalmol 1987; 105: 1486-7.

24 Carter CB. Hamill RJ, Matoba AY. Syphilitic optic neuritis in a patient with a positive test for AIDS. Arch Ophthalmo 1987; 105: 1485-6.

25 Hicks CB, Benson PM, Lupton GP, Tramont EC. Seronegative secondary syphilis in a patient infected with the human immunodeficiency virus with Kaposi sarcoma. Ann Intern Med 1987; 107: 492-5.

26 Macher A, Rodrigues M, Laplan W, et al. Disseminated bilateral chorioretinitis due to Histoplasma capsulatum in a patient with the acquired imm
27 Gass JD, Sever RJ, Grizzard SW, et al. Multifocal pigment epithelial

28 Davis JL Nussenblatt RB, Bachman DM. Endogenous bacterial retinitis in AIDS. Am 7 Ophthalmol 1989; 107: 613-23.

29 Marcus DF, Bovino JA, Burton TC. Sarcoid granuloma of the choroid. Ophthalmology 1982; 89: 1326-30.

30 Campo RV, Aaberg TM. Choroidal granuloma in sarcoidosis Am F Ophthalmol 1984; 97: 419-27.

31 Gass JDM, Olson CL. Sarcoidosis with optic nerve and retinal Gass JDM, Olson CL. Sarcoidosis with optic nerve
involvement. Arch Ophthalmol 1976; 94: 945-50.

32 involvement. Arch Ophthalmol 1976; 94: 945-50. acute infection model for Mycobacterium intracellulare disease using beige mice. Am Res Respir Dis 1983; 127: 648-9. 